\title{
NEUTRON AND X-RAY SCATTERING IN THE STUDIES OF METALS
}

\author{
L. DOBRZYŃSKI ${ }^{a, b, c}$ \\ ${ }^{a}$ Institute of Physics, University in Białystok, Lipowa 41, 15-424 Białystok, Poland \\ ${ }^{b}$ The Soltan Institute for Nuclear Studies, 05-400 Otwock-Świerk, Poland \\ ${ }^{c}$ Laboratoire de Minéralogie-Cristallographie, Université de Paris VI \\ 4 place Jussieu, 75252 Paris Cedex 05, France
}

Neutrons and X-rays are well established tools for studying properties of condensed matter. With the advent of synchrotron radiation facilities their high photon fluxes started to be effectively used in the fields reserved earlier for neutrons. Although the two techniques are conceptually so similar that many physical problems can be solved using either technique, there are apparent fields in which one method is superior with respect to the other one. Therefore both techniques are often needed for characterization of the material of interest. The paper concentrates on the studies of some static properties of metals and alloys. Relatively wide range of phenomena is presented, starting from structural aspects and ending on the sophisticated studies of the magnetic moment components. The paper is intended to bring a short (yet necessarily subjective and incomplete) review of what has recently been achieved by using neutrons and X-rays in metal (or more precisely - metallic states) studies.

PACS numbers: 71.55.Ak, 25.40.Dn, 41.60.Ap

\section{Introduction}

To make any serious review which would present the results of studies of metals by neutron and X-ray scattering requires a book and perhaps a single volume would not be sufficient. Therefore we will only sketch some of the studies undertaken in last years and try to select experiments which were delivering either complementary information or aimed at unique features possible to observe using one of the two methods only. We will also limit our interest primarily to microscopic phenomena, therefore e.g. metallurgical aspects of metals will not be covered. We will focus mainly on the static aspects, therefore the elementary excitations will be mentioned very briefly only. Even assuming such strong limitations, we are afraid that the choice of examples will be very incomplete and very subjective. But, let us try... 


\section{Different probes, different basic interactions, complementarity of the techniques}

Community of solid state physicists knows that neutrons and $\mathrm{X}$-rays are complementary tools of studying the structure of solids. This follows from simple considerations of the conditions which a given probe should fulfil in order to carry out such studies. In fact one only needs to have sufficiently intensive radiation with the wavelength comparable to the one typical of a solid, i.e. of the order of 1 or several $\AA$. Typical X-rays obtained from a classical X-ray tube fulfil this condition (the wavelength of $1 \AA$ is achieved with the $12.4 \mathrm{keV}$ energy), and thermal neutrons from either stationary or pulsed sources deliver neutrons with similar wavelength (the neutron energy must be equal in this case to about $80 \mathrm{meV}$ ).

The principal interaction of X-rays is governed by electromagnetic forces, the interaction of the radiation with electronic charges in matter is strong, which results in rather heavy absorption of this radiation. On the contrary, the principal neutron interaction is going through nuclear forces, it is relatively weak, and therefore neutrons penetrate relative large volumes of substances under study. In order to compensate for this weakness, and relatively low available neutron fluxes with comparison to the X-ray ones, the volumes of the samples used in neutron diffraction have to be orders of magnitude larger than the volumes sufficient for X-ray studies. On the other hand, the interaction strength as measured by the scattering length varies linearly with the number of electrons in an atom in the case of X-ray scattering, while there is no correlation between the neutron scattering length and the atomic number of the element. Moreover, the isotopes of the same elements can have vastly different neutron scattering lengths with even different sign. These features are very important for obtaining good "optical contrast" between various elements. Therefore a deficiency connected with lower neutron fluxes may be compensated by much better visibility of given elements on the background of the other ones. When the structural aspects are concerned, none of the techniques is truly superior with respect to the other.

There is one type of interaction which made neutrons very unique probe in solid state physics: we mean here magnetic interaction of neutrons with the magnetic spins and currents in matter. The electrically neutral neutron has a magnetic moment, interaction of which with an atomic spin may lead to the scattering amplitude larger than or comparable with the nuclear one. Therefore neutron moment senses spatial variations of the magnetic induction within the body and can bring information on the magnetic structure of solids. Using neutrons we may thus get not only information on "where the atoms are" (Nobel Prize for C.G. Shull) but also where, how large and how oriented magnetic moments exist in the lattice.

Of course, the neutron magnetic and nuclear interactions take place at the same time, therefore sometimes the effects of these interactions overlap. Fortunately, neutron physicists learned to make use of this overlap and produce polarized neutron beams. These beams are of invaluable use whenever one has to separate magnetic from non-magnetic scattering.

Magnetic interaction is also natural for X-rays: after all, the electromagnetic wave has not only electric, but also magnetic component. The interaction of this 
component with internal magnetizations is very weak and hardly visible. The situation is not, however, hopeless. With the advent of synchrotron radiation facilities, the fluxes of photons delivered to the samples are so high that the measurements of even such weak magnetic effects became possible. The first experiments [1] were conducted as early as in 1972 on antiferromagnets for which the effects of the magnetic structure are not mixed with the scattering on the crystallochemical structure. With time it was discovered [2] that when the radiation has an energy close to the absorption edge of a magnetic moment possessing element, one can obtain huge enhancement of the signal connected with this moment also for the ferro- or ferrimagnetic case. This discovery of the late 80 -ies was truly sensational: for the first time it became possible to study the magnetic structure within a sublattice of given type of atoms. And in addition, X-rays brought as a rule much improved resolution in the momentum space. Therefore, although still not being fully competitive to neutrons, they started to deliver often unique, complementary information which could hardly be obtained by neutrons alone. In particular, studies of thin films, including surface physics problems, or critical scattering from small volume samples may be mentioned. An interesting counterpart of the magnetic neutron diffraction is the magnetic X-ray diffraction. The magnetic X-ray scattering amplitude (away from resonances) for an atom is given by [3]

$$
f(q)=-\mathrm{i}\left(E / m c^{2}\right)[0.5 \boldsymbol{L}(\boldsymbol{q}) \boldsymbol{A}+\boldsymbol{S}(q) \boldsymbol{B}],
$$

where $E$ denotes the photon energy, $\boldsymbol{L}(q)$ and $\boldsymbol{S}(q)$ are Fourier transforms of the orbital and spin magnetic moment, respectively, vector $q$ denotes the momentum transfer of the photon (equal in the case of the Bragg scattering to one of the reciprocal lattice vectors), and vectors $\boldsymbol{A}$ and $\boldsymbol{B}$, which follow from the way the photon magnetic field couples to the magnetization express the photon polarization dependencies and have different angular dependencies. This last feature, used recently e.g. in studies [4], enables one to separate out orbital from spin scattering once the magnetic scattering is observed. We recall that in the case of the magnetic neutron scattering a similar expression contains a simple sum of $0.5 L(q)+S(q)$, therefore the separation is possible only under certain assumptions concerning the $q$-dependence of spin and orbital parts of the magnetic scattering amplitude.

The expression (1) will not be valid when the photon energy is tuned close to the resonance. A huge enhancement of the magnetic signal is then observed, which enables to study elemental magnetism in detail which are hard to observe by neutrons. Indeed, a few orders of magnitude enhancement of the magnetic signal at the $M_{\mathrm{IV}}$ and $M_{\mathrm{V}}$ edges in actinides allows for measurements of extremely small ordered magnetic moments. In fact, the moments as small as $0.02 \mu_{\mathrm{B}}$ were measured [5]. It should be noted here, however, that the resonant scattering depends on the total magnetic moment and the magnetic form factor is not present in respective formulas. The separation of the moment into the $\boldsymbol{L}$ and $\boldsymbol{S}$ components is thus not easily achievable. The resonant scattering is not only element specific. It is also orbital specific, so e.g. itinerant $5 d$ and localised $4 f$ states in rare-earth metals can be probed independently, see e.g. [6]. This paper aims in solving magnetic structure of Ho-Pr alloys and follows earlier neutron studies [7], thus constituting very good examples of how both techniques can be used complementarily. 
In spite of many illuminating examples existing in the literature, the physical information on the magnetism brought by magnetic X-ray scattering is more rare than the one delivered by the magnetic neutron scattering. Rather elementary studies of magnetic structures by the neutron powder diffraction technique can hardly be done with X-rays. This may change in the future, as it seems that one can forecast the development of synchrotrons with increased photon fluxes whereas possibilities of a similar development of the neutron sources are less bright.

The element-specificity plays now major role in many kinds of experiments performed with X-rays. It also turned out that near resonances (absorption edges), the circularly polarized X-rays exhibit magnetic dichroism, i.e. their absorption depends on a mutual orientation of the atomic spin and the helicity of the polarization. Magnetic circular dichroism of X-rays (XMCD) is a truly unique effect of great beauty.

Having strong neutron and X-ray sources the sample volume problems are only in rare cases giving apparent advantage to one of the methods. If one leaves aside pathological cases of highly neutron absorbing samples (e.g. the ones containing gadolinium or samarium) or samples with extra small volumes, which are inaccessible for neutron studies, it is much more important now what physical information may be delivered for the same sample by these two techniques. There are fields in which neutrons are still unbeatable because of another probe characteristics which was not exploited till now, namely the energy. The energy of thermal neutrons with the wavelength of the order of a few $\AA$ is in the range of about $5-100 \mathrm{meV}$. This range is ideally suited to study elementary excitations in the crystal and magnetic lattices, in which the energies of elementary excitations are just within this energy band. Using the so-called cold neutron source one can substantially increase the neutron flux at much lower energies and make it possible to study excitation in the meV range. On the contrary, using hot neutron source, the energy range excitations which are possible to study increase to the electronovolt range. Neutrons are ideal for studying interatomic correlations of both, nonmagnetic and magnetic origins, fluctuations in the system, and even electronic transitions. And neutrons are truly non-destructive probe. How not to love them?

There exist many excellent tutorial books on neutrons and X-rays. For getting good general overview of both techniques we would recommend 3 volumes published after HERCULES course [8]. In the following we shall try to show just a few examples of experiments which demonstrate powerfulness of these methods applied to the studies of metals. The details of the experiments will be explained whenever necessary. We shall start with two examples in which, for the time being, only one technique can bring high quality results. Next, we will present the experiments in which both tools are or may be used in a complementary way.

\section{Antiferromagnetism of fcc iron and anti-invar effect in fcc $\mathrm{Fe}-\mathrm{Ni}$ alloys}

We start with the neutron experiment on iron-rich $\mathrm{Fe}-\mathrm{Ni}$ alloys which ex-. hibit a volume enhancement in the paramagnetic phase and its volume expansion coefficient is relatively large $[9,10]$. This so-called anti-invar effect is known also 
for pure fcc Fe and $\mathrm{Mn}$ metals; the invar effect is observed for alloys only. Although the ground state of fcc $\mathrm{Fe}$ and $\mathrm{Mn}$ is antiferromagnetic, the energy of ferromagnetic states is not much higher. It is sufficient to expand the volume by $5 \%$ in order to make the ferromagnetic state stable. The magnetovolume instabilities in $\mathrm{Fe}$ and $\mathrm{Mn}$ were treated theoretically in [11]. It is interesting to note that the magnetic moment is continuously increasing with increasing volume when in the antiferromagnetic state. Were the fcc Fe ferromagnetic, a discontinuous transition from a zero-moment state to the high-spin state (with the moment of about $2 \mu_{\mathrm{B}}$ ) would take place.

The fcc $\mathrm{Fe}_{100-x} \mathrm{Ni}_{x}$ alloys are ferromagnetic for $x$ above about 20. If the alloys were fcc below this concentration (they are bcc), one could speculate on their antiferromagnetism with the Néel temperature gradually increasing with decreasing nickel content. The neutron studies [9] concerned magnetic correlations in paramagnetic phase. Because the discrimination of the magnetic scattering from the non-magnetic one was of vital importance, the use of polarised neutrons and polarisation analysis of the scattered neutrons became necessary. Figure 1 shows the obtained dependence of the scattering cross-section on the momentum transfer and temperature for alloys with different compositions. Firstly, apparent forward scattering seen in all cases unambiguously indicates presence of ferromagnetic (short-range) correlations. Such correlations are to be expected for the samples with $x$ larger than 20 . For samples with $x=15$ and $x=10$, however, antiferromagnetic correlations were expected. They should manifest through the appearance of a diffuse peak around positions of (100) and (110) peaks, i.e. at the momentum transfer $q$ of about $1.7 \AA^{-1}$ and $2.5 \AA^{-1}$, respectively. Certainly nothing like that is observed. Secondly, the scattering cross-section at small $q$ 's for the ferromagnetic samples decreases with increasing temperature, which is expected (the ferromagnetic correlations become weaker). The cross-section for $x=15$ is almost temperature-independent and for $x=10$ it increases (!) with increasing temperature. Thirdly, if the $q$-dependence is analysed quantitatively, one can show that the spin-spin correlation range decreases with increasing temperature for $x$ down to 20 , whereas for $x=15$ and $x=10$ it is almost temperature independent and stays on the level of about $30 \AA^{-1}$. In fact similar value was obtained for fcc $\mathrm{Fe}$ above $1300^{\circ} \mathrm{C}$.

Taking the results for $x=35$ as the reference point (this composition is typical of invar alloy, therefore no anti-invar effects can show up in it), the simplest way of understanding the results obtained for the anti-invar alloy $x=10$ is that the sample undergoes a transition from the nonmagnetic to the ferromagnetic state upon increasing temperature. The magnetic correlations connected with this transition must overweight "normal" decay of ferromagnetic correlations with increasing temperature (observed e.g. for the sample $x=35$ ). With increasing $\mathrm{Ni}$ content the volume expansion becomes weaker due to the anti-invar behaviour and consistently, the effect of the nonmagnetic-high-spin magnetic transition is diminished. Apparently both counteracting effects compensate in the vicinity of $x=15$.

The fact that in spite of expectations no antiferromagnetic correlations were found does not mean that they are not present. The expected Néel temperature 

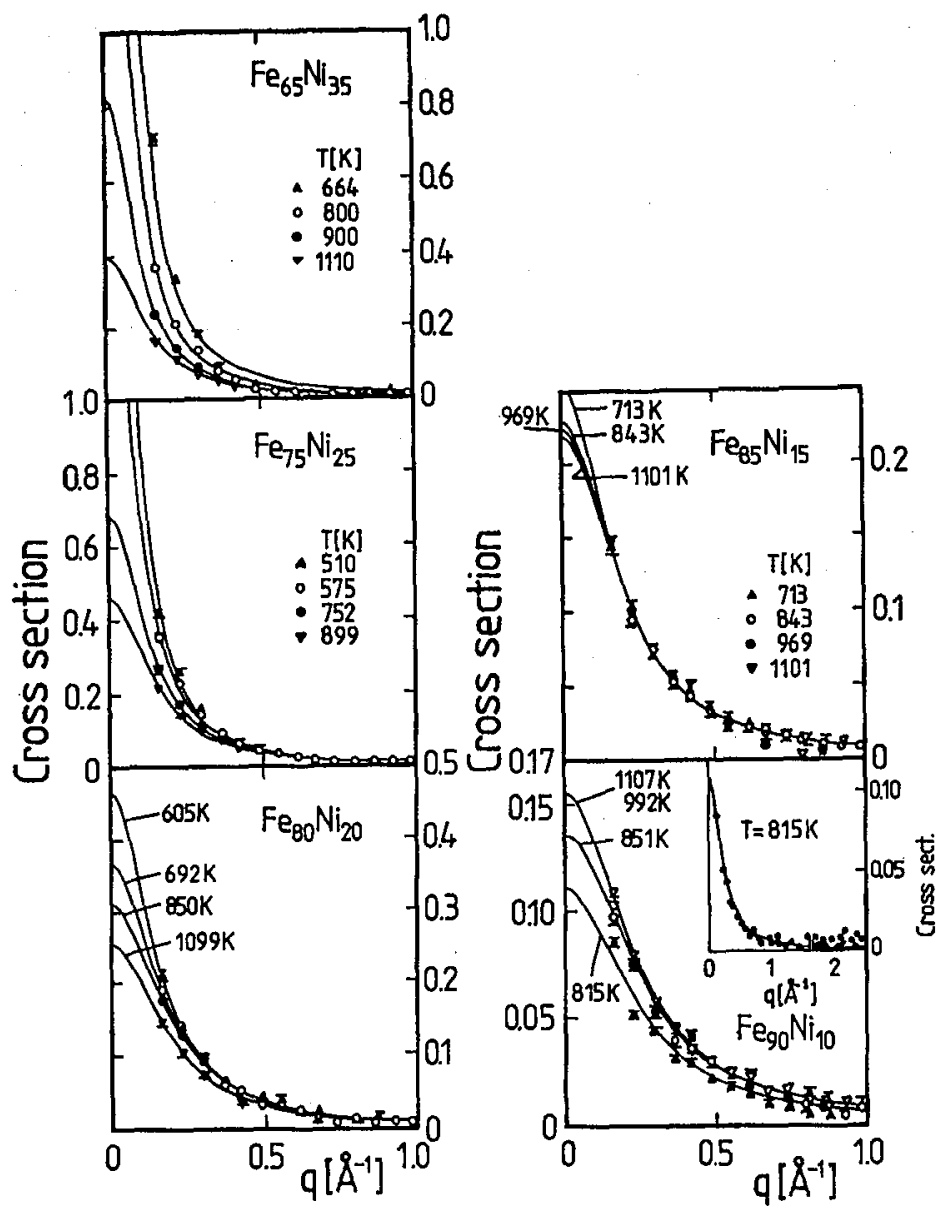

Fig. 1. Paramagnetic scattering cross-section (in barn/steradian) versus momentum transfer for various temperatures and sample compositions for fcc Fe-Ni alloys. Reproduced with permission from Ref. [9]. Copyright by EDP Sciences.

(about $65 \mathrm{~K}$ ) is much lower than the temperature range covered by the experiment. Therefore it may well be that the ferromagnetic correlations at higher temperatures dominate the antiferromagnetic ones. Unfortunately, making the experiment at much lower temperatures has no sense because the sample structure would transform into the bcc type, in which the magnetovolume effects also change.

\section{Magnetism of ultrathin films of $\mathrm{Mn}$}

The problem of magnetism of $\mathrm{Mn}$ on and close to the surface falls into the same category as the previous example. Going from the bulk to the surface means changing the volume available for an atom. In ultrathin layers this volume may be regulated somehow by a substrate on which the film is grown. The cited experiments concern the layers of Mn grown on (001) bcc Fe [12] and (001) fcc Co [13]. Their magnetic properties shed some light on the magnetic $\mathrm{Mn}-\mathrm{Mn}, \mathrm{Fe}-\mathrm{Mn}$ and 

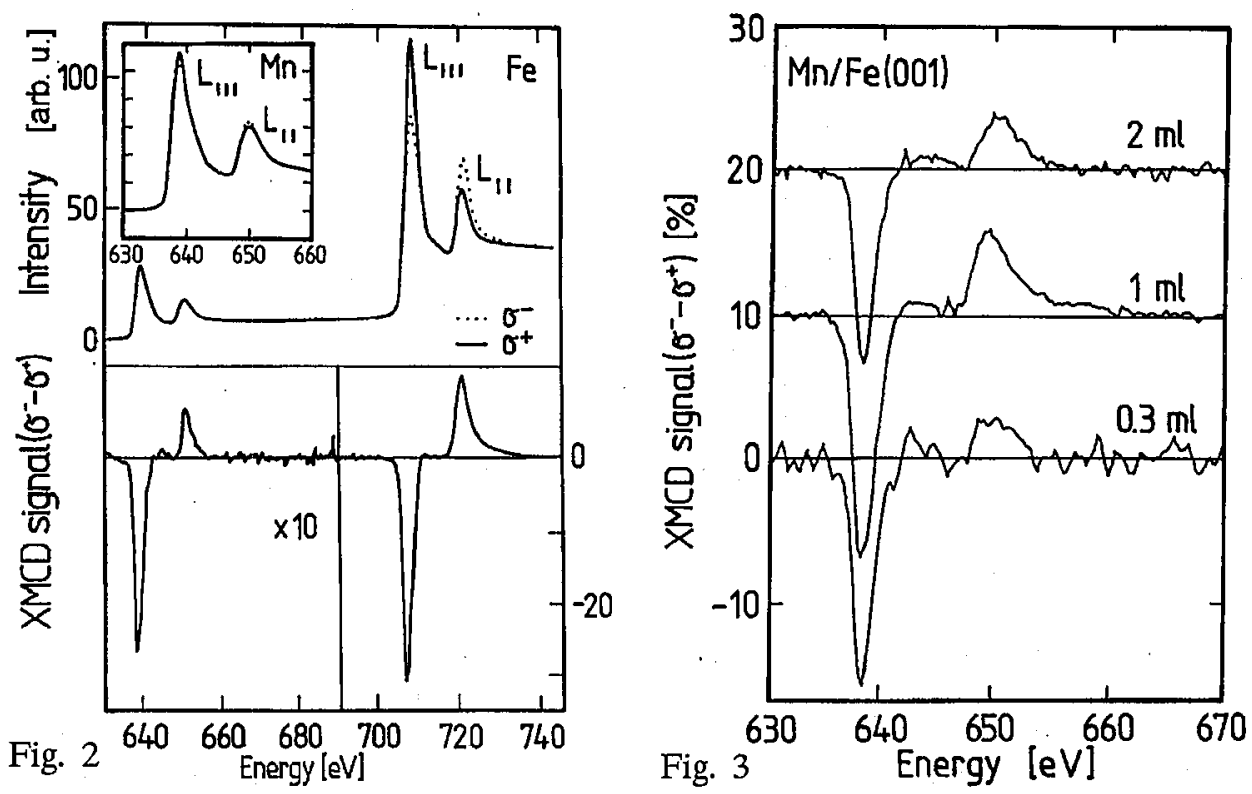

Fig. 2. XMCD signals measured for $\mathrm{Fe}$ and $\mathrm{Mn}$ at $L_{\mathrm{II}}$ and $L_{\mathrm{III}}$ absorption edges for one monolayer of $\mathrm{Mn}$ grown on (100) bcc Fe. Continuous and dashed lines in the upper figures show direct results obtained with two opposite directions of the magnetic field with respect to the incoming circularly polarized radiation. Reproduced with permission from Ref. [12]. Copyright by EDP Sciences.

Fig. 3. XMCD spectra observed at room temperature at $\mathrm{Mn} L_{\mathrm{II}}$ and $L_{\mathrm{III}}$ edges as a function of the manganese layer thickness. Reproduced with permission from Ref. [12]. Copyright by EDP Sciences.

Co-Mn interactions. Typically, $\mathrm{Mn}$ is always found to be antiferromagnetic in the bulk, and it is often believed that the Fe-Mn exchange interaction should be of the antiferromagnetic nature. Therefore the studies of ultrathin films of $\mathrm{Mn}$, where the volume given to $\mathrm{Mn}$ is much expanded, were of great interest.

In the case of $\mathrm{Mn}$ grown on Fe the literature data, reviewed in [12], are highly confusing. The authors were thus very careful when growing the films (by $\mathrm{MBE}$ technique) and carrying out their detailed characterisations prior to making the experiment. Figure 2 shows the XMCD spectra obtained for one atomic layer at Fe and $\mathrm{Mn} L_{\mathrm{II}}$ and $L_{\mathrm{III}}$ absorption edges. No doubt, manganese acquires magnetic moment. Moreover, contrary to some theoretical expectations and experimental results (see citations in [12]) dichroism of both elements looks much the same, which means that iron and manganese magnetic moments are parallel. No qualitative changes of the spectra were observed when the number of $\mathrm{Mn}$ monolayers was gradually increasing to 2, see Fig. 3 . This means that the second monolayer has the magnetic moments parallel to the first one. The two Mn layers are coupled. ferromagnetically! When the manganese moment is concerned, the authors conclude that it is $1.75 \mu_{\mathrm{B}}$ /atom for a monolayer and, on the average, it drops down to $1.4 \mu_{\mathrm{B}}$ /atom in the case of two monolayers. 


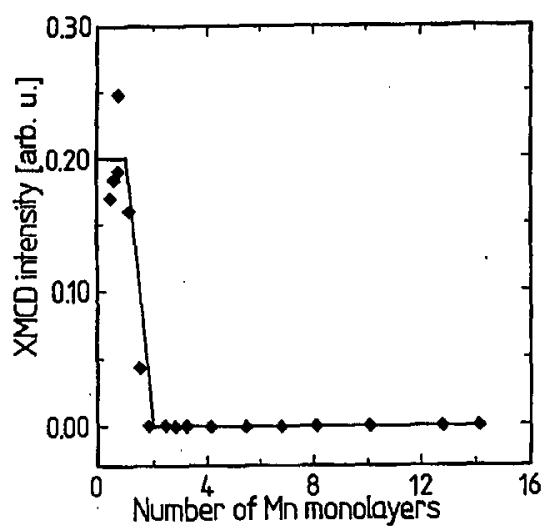

Fig. 4. XMCD signal at Mn $L_{I I I}$ edge as a function of the number of $\mathrm{Mn}$ monolayers grown on (100) fcc Co (after [13]).

In the case of Mn layers on (001) fcc Co [13] the magnetic moment of manganese on cobalt substrate turns out to be enhanced. The ferromagnetic cobalt substrate is inducing also ferromagnetic ordering within the manganese monolayer. However, the XMCD experiment [13] shows that the ordering disappears already at two monolayers. How abrupt the change from magnetic order to a nonmagnetic behaviour is may be judged from Fig. 4 which shows the value of the XMCD signal at $L_{\text {III }}$ edge. The way the magnetic order disappears shows that the manganese layer becomes ferromagnetic when cobalt is its only neighbour. When extra Mn layer covers the first one, the magnetic order disappears (we recall that in the case of iron substrate the moment decreases but does not disappear). Of course, the magnetic moments of $\mathrm{Mn}$ do not disappear. As the authors of [8] show, the first monolayer has enhanced magnetic moment, which is quickly decreasing to the value of the bulk magnetic moment when the number of monolayers grow. Therefore the disappearance of the ferromagnetic order must be due to the competing $\mathrm{Co}-\mathrm{Mn}$ and $\mathrm{Mn}-\mathrm{Mn}$ interactions.

\section{Are all atoms sitting at lattice sites determined by the crystal structure?}

It is well known that there are many alloys which do not follow Vegard's law. This is the case of some $\mathrm{Ni}-\mathrm{Fe}$ alloys whose lattice parameter increases unexpectedly fast with iron content. If an alloy is disordered, how can we be sure that the interatomic distances are always given by the overall lattice parameters? One can imagine that in the case of $A B$ alloys the average distances between AA and $\mathrm{BB}$ pairs may be somewhat different and $\mathrm{AB}$ distances only will be uniquely determined by the lattice parameter. This is important because the atomic displacements can play some role in the total crystal energy which was theoretically recognized some time ago [14]. Convincing and, to my opinion, beautiful studies have been published by Jiang in 1996 [15] who studied an atomic order in single crystals of $\mathrm{Fe}_{46.5} \mathrm{Ni}_{53.5}$ and $\mathrm{Fe}_{22.5} \mathrm{Ni}_{77.5}$ by means of $\mathrm{X}$-ray diffuse scattering carried out with the use of three different X-ray wavelengths at the National Synchrotron 
Light Source at the Brookhaven National Laboratory, USA. The choice of particular wavelengths was aimed at ensuring either the highest or the lowest optical contrast, or at maximizing the importance of an interference term which appears in a rather lengthy formula for the diffuse scattering. As in XMCD, the resonant interactions were used for changing the optical contrasts. What is a novelty here, however, is extremely high precision of the diffuse scattering studies. It allowed one for observing the effects arising not only from the chemical disorder, but also from displacements of the atomic positions. It is worth mentioning that having an alloy consisting of $\mathrm{Fe}$ and $\mathrm{Ni}$ atoms, which are lying close to each other in the periodic table of elements, makes the X-ray experiment extremely difficult. Therefore for this kind of alloys it was always much easier to use neutrons and study diffuse scattering from a sample in which nickel was enriched in the isotope ${ }^{62} \mathrm{Ni}$. Such a substitution was ensuring large contrast between iron and nickel, therefore the determination of the Cowley-Warren short-range order parameters was quite straightforward (which does not mean - simple).

A knowledge of the displacements in disordered alloys is interested in itself. It may also be very important for understanding the magnetic properties e.g. such ones as discussed by us in Sec. 3. By the displacement is meant a set of $\delta_{p q}$ values, see Fig. 5. It is important to realize that the well known EXAFS method is not

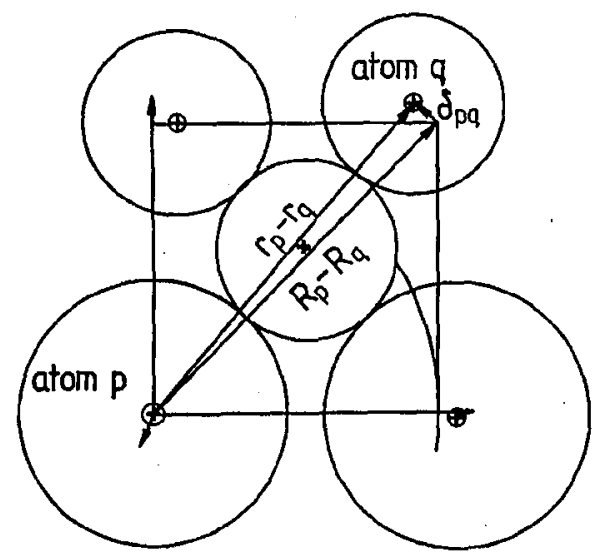

Fig. 5

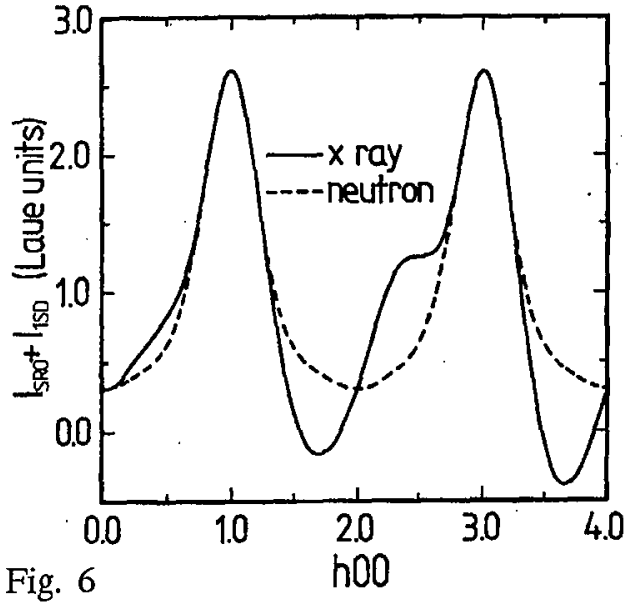

Fig. 5. Schematic picture showing that instead of expected lattice vector separating $p$ and $q$ species, $\boldsymbol{R}_{p q}=\boldsymbol{R}_{p}-\boldsymbol{R}_{q}$, the atom, say $p$, can be shifted by $\delta_{p q}$. Of course, the weighted average of all the displacements (for any atomic pairs) must be zero.

Fig. 6. Comparison of the diffuse intensity measured by X-rays (resonant $7092 \mathrm{eV}$ ) and neutrons for the $\mathrm{Fe}_{23.5} \mathrm{Ni}_{76.5}$ quenched from $685^{\circ} \mathrm{C}$ (after [15]). The neutron intensity for a sample in which $\mathrm{Ni}$ was $97.7 \%$ enriched in ${ }^{62} \mathrm{Ni}$ was simulated taking the short-range order Cowley-Warren parameters from the original neutron experiment and static displacements from the $\mathrm{X}$-ray measurements described in the text. Only first two terms appearing in the cross-section are calculated, which explains why negative values may appear. The asymmetry of X-ray curve is clearly associated with the static displacements, which are invisible for neutrons. 
suitable for determination of $\delta_{p q}$ 's because its spatial resolution allows one for determination of the distances with accuracies of the order of $0.01 \AA$ only, whereas the displacements may be smaller than $0.001 \AA$. Figure 6 shows [15] that a similar neutron experiment on isotopically substituted samples would not perform better: the static displacements leading to extra features observed in the X-ray scattering would not be seen by neutrons.

The most essential conclusion of the paper [15] is that the nearest neighbour iron-iron displacement in the alloys under study is large, $0.03-0.05 \AA$, yet the average distance between $\mathrm{Fe}-\mathrm{Fe}$ pairs along $\langle 110\rangle$ direction is not sensitive to the alloy composition and equals to about $2.563 \AA$. The average separation of the second $\mathrm{Fe}-\mathrm{Fe}$ nearest neighbours is, on the other hand, reduced. The Ni-Ni distance diminishes from 2.533 to $2.511 \AA$ when the nickel content increases. Similar contraction of the distances, from 2.521 to $2.508 \AA$, is observed between $\mathrm{Fe}-\mathrm{Ni}$ pairs. Thus the much enhanced (with respect to the expectations based on the Vegard law) volume expansion when iron is added to nickel follows from large distances between $\mathrm{Fe}-\mathrm{Fe}$ pairs, and increasing distances between $\mathrm{Ni}-\mathrm{Ni}$ and $\mathrm{Ni}-\mathrm{Fe}$ pairs. It should be stressed that it is not the larger volume of iron atoms which makes this effect. In fact, when one calculates the radii of nickel and iron atoms (from their average distances to 12 nearest neighbours), the radius of iron atom does not differ by more than $0.2 \%$ of that one expected from the unit-cell dimensions. What is found is a property of the bonds! This in turn calls for detailed studies of the electronic structure, charge transfer and charge buildup effects, as well as for rethinking the problem of magnetism of these alloys.

\section{What's going on during order-disorder transition?}

The order-disorder transitions are common for alloys. There are some which exhibit a sequence of such transitions. For example, $\mathrm{Fe}_{3} \mathrm{Si}$ and $\mathrm{Fe}_{3} \mathrm{Al}$ alloys change their structure upon lowering temperature from bcc (A2) to B2 and finally to $\mathrm{DO}_{3}$ type structure. It is generally interesting to know kinetics of the atomic ordering from one to another crystallographic pattern, especially because one can hardly imagine that the ordering takes place instantaneously. A huge amount of photons available at the synchrotron sources plus coherency of the beams from insertion devices (the so-called undulators) made it possible to visualise the process of the ordering. In elegant experiments $[16,17]$ the $\mathrm{B} 2-\mathrm{DO}_{3}$ phase transition in $\mathrm{Fe}_{3} \mathrm{Al}$ was studied. The very basis of the experimental idea consists in the fact that when coherent light is scattered from a disordered system it gives rise to a random diffraction or so-called "specle"-pattern. Due to the coherent beam used, this pattern is showing exact spatial arrangement in the system (scattering amplitudes add within the region comparable with the coherence length of the beam); when an incoherent beam is used, the scattering pattern reflects only the probability distribution of the disorder (intensities add). If the spatial arrangement evolves with time, the observation of evolving specle pattern brings thus information on the dynamics of the system. In particular, at every single point in a specle the intensity fluctuation should be observed.

A characteristic signature of the $\mathrm{DO}_{3}$ phase is the appearance of the (111) peak, which is forbidden in B2 structure. The crystal was thus set at the Bragg 
position for the (111) reflection and the impinging beam was tailored by a $4 \mu \mathrm{m}$ pinhole ensuring coherency of the transmitted beam in horizontal as well as vertical directions. The scattered beam was measured by a position sensitive detector or CCD camera with $20 \mu \mathrm{m}$ spatial resolution [17]. Position and arrival time of every photon could be recorded. The observed ordered domain size turns out to be about $1200 \AA$. Above the phase transition temperature the specle intensity fluctuates. with time which in turn brings information on the time correlation function in the system studied. The cited experiments open up a new field of investigating dynamics of equilibrium critical fluctuations.

\section{Magnetic moments and their components}

Decomposition of the individual moments into the spin and orbital components in an alloy is not a trivial task. In addition to the $d$-or $f$-type moment, the conduction band can also be polarised and contributes to the resultant moment. A typical neutron experiment in which such problems are considered consists in measuring the magnetic form factor by means of the polarised neutron diffraction and applying either a model or carrying out accurate Fourier analysis. The description of such experiments can be found in most of the standard text books on neutron diffraction. Unfortunately, in order to arrive at a conclusion one must use assumptions which are not always certain enough. Moreover, in the case of multicomponent, disordered alloys, it is often impossible to separate contributions coming from different atoms, so the aforementioned XMCD becomes a technique of choice.

We want to quote an example taken from probably one of the best introductory text on XMCD due to Schütz et al. [18]. Figure 7 shows a sketch of the absorption profiles expected in the vicinity of $L_{\text {II }}$ and $L_{\text {III }}$ edges for an atom with partly only filled $d$-shell. Whether the dichroic signal is positive or negative depends on the mutual orientation of the atomic spin with respect to the beam incoming direction and helicity of the beam (we recall that the magnetic phenomena are observed for circularly polarised beams only; we limit our attention to ferromagnets). Because the whole process is taking place with emission of a photoelectron and its orbital quantum number can change in discrete manner only, this electron carries also information about the initial orbital state or orbital moment $L$. Figure 7 shows expectations when the magnetic moment is of spin-only or orbital-only type, the latter assumed to be oriented antiparallel to the photon helicity (unlikely case). Depending on the $S-L$ coupling the resultant magnetic dichroic signal must be thus either enhanced or depressed. Playing with some combinations of absorption coefficients differences one can obtain spin and orbital moments independently. Of course, in practice, extraction of the orbital and spin moments is not always so straightforward and needs some carefulness.

An example of the application of this method can be found in solving a problem of how platinum atoms are polarised by neighbouring Co atoms in the multilayer system Co/Pt [18]. It is well known that cobalt is ferromagnetic. The question is, how this ferromagnetism is carried through the non-ferromagnetic medium, platinum in this case. The sensitivity of XMCD technique to the element under study allows one to use ingenious experimental trick, namely using 


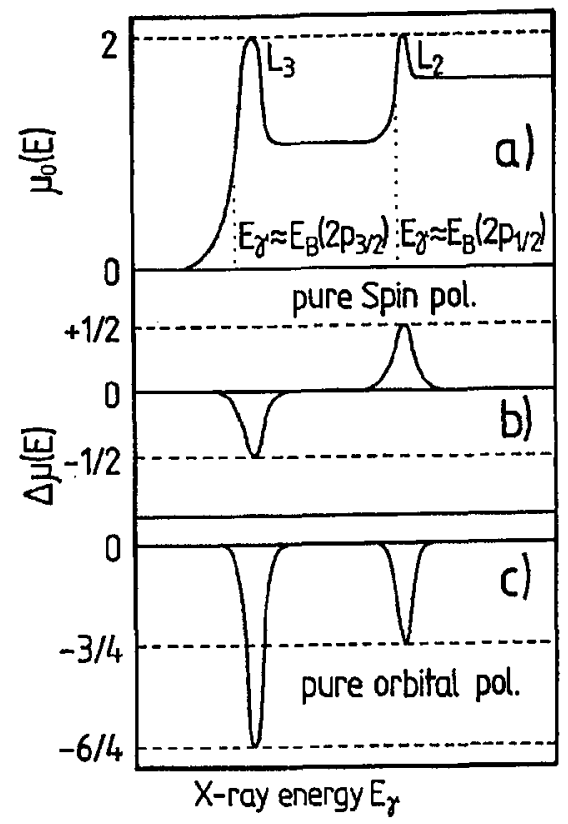

Fig. 7. Absorption coefficients and $L_{\mathrm{II}}$ and $L_{\mathrm{III}}$ edges for an atom with unfilled $d$-shell (top). Change of the absorption coefficient when the relative orientation of atomic spin and photon helicity reverses and the atom has spin-only magnetic moment (middle part). XMCD signal for orbital-only final state (bottom). Reproduced with permission from Ref. [18]. Copyright by Gordon and Breach Publishers.

iridium layer buried within platinum ones. Iridium response to the ferromagnetic nearest neighbour is similar to the one of platinum atom, therefore presence of iridium should not affect the overall properties of the multilayer system under study. Studying such an effect layer by layer one arrives at the result displayed in Fig. 8, which shows that both, spin and orbital, magnetic moments of platinum and iridium could be measured. The presented results disprove some theoretical expectations concerning the magnetization distribution in platinum layers.

The XMCD technique was used for studies of $3 d$ metals by O'Brien et al. [19]. Their results are shown in Fig. 9 in normal (left-hand side) and expanded (right-hand side) scales. In addition to the successful discrimination of the orbital moment from the spin one, particular attention was paid to the presence of a shoulder seen in $\mathrm{Ni}$ and Co spectra (between the abbreviations $\mathrm{B}$ and $\mathrm{L}_{2}$ ). This shoulder is due to the presence of a polarization of the conduction band. That such a polarization should appear is expected from various theoretical approaches, to quote one of the latest of Eriksson et al. [20]. The corresponding diffuse magnetic moment is negative and - according to [20] - on the level of $2 \%$ (in $\mathrm{Fe}$ ), $4 \%(\mathrm{Co}$ ) and $7 \%$ (nickel). On the other hand, the diffuse moments, determined in early polarised neutron Bragg diffraction studies, were much higher and amounted to $10 \%, 16 \%$ and $17 \%$, respectively. A review of this problem for metals and alloys can be found e.g. in Ref. [21]. In quite recent analysis of the neutron magnetic form factors 

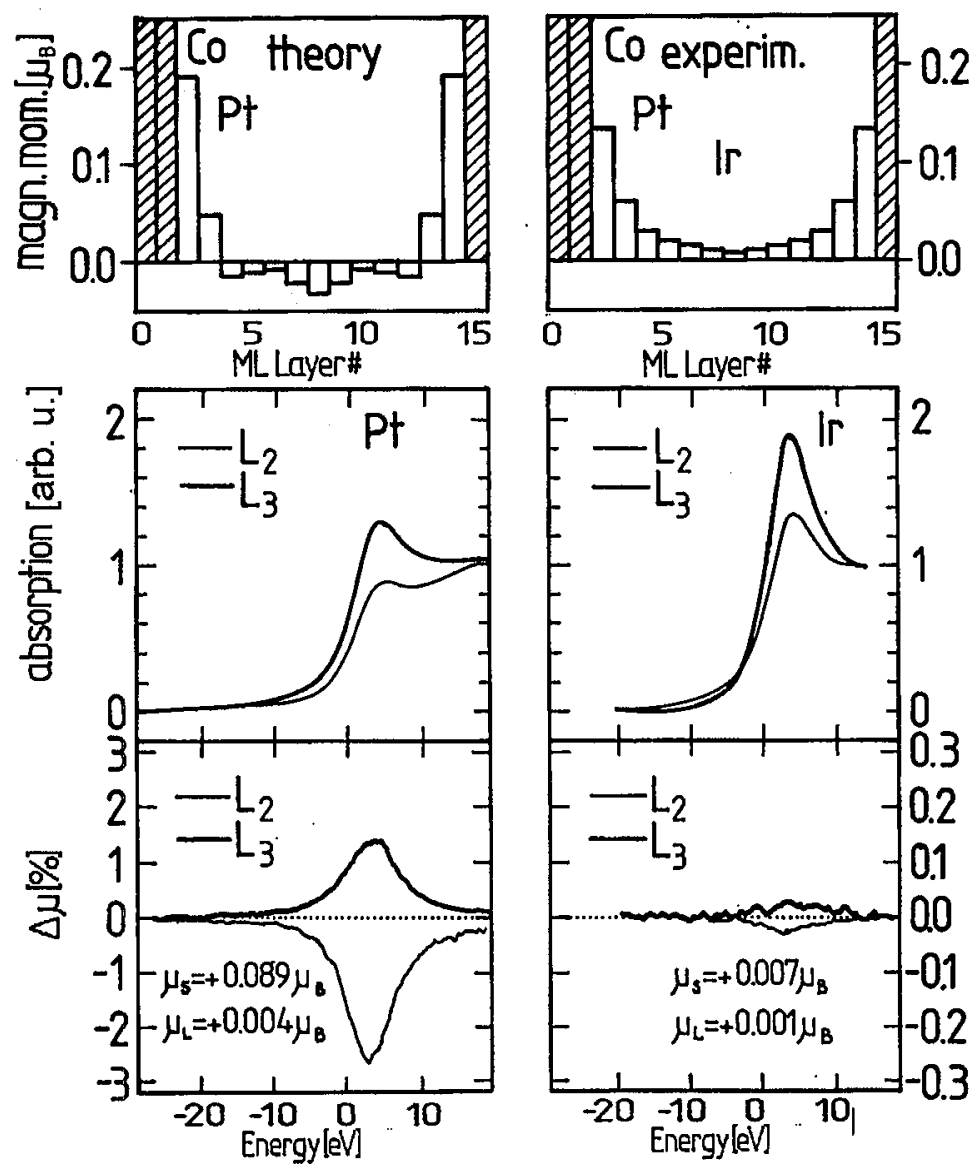

Fig. 8. Results of the XMCD studies of $2 \mathrm{ML} \mathrm{Co} / 13 \mathrm{ML}$ Pt multilayered system. Reproduced with permission from Ref. [18]. Copyright by Gordon and Breach Publishers.

by means of the maximum entropy method [22] the conclusion was that neutron results alone bring not enough evidence for stating the presence of any uniform negative polarization. The interpretation of the small shoulder in XMCD spectra may also seem not too convincing in this respect.

In this situation we should like to turn attention to a group of completely different X-ray experiments connected with the Compton scattering. A projection of electron momentum density on a given crystallographic direction is measured in these experiments [23]. This quantity (which is a function of the ejected electron momentum component along the measured direction) is called the Compton profile. Because of the very nature of the interaction, the probe is much more sensitive to external $(s, p)$ electrons than to the more internal, $3 d$, ones. Using circularly polarised photons and switching either their helicity or the magnetization of a ferromagnetic sample one can measure, through a difference of the Compton profiles (called magnetic Compton profile) the magnetic contribution to the scattering. It 

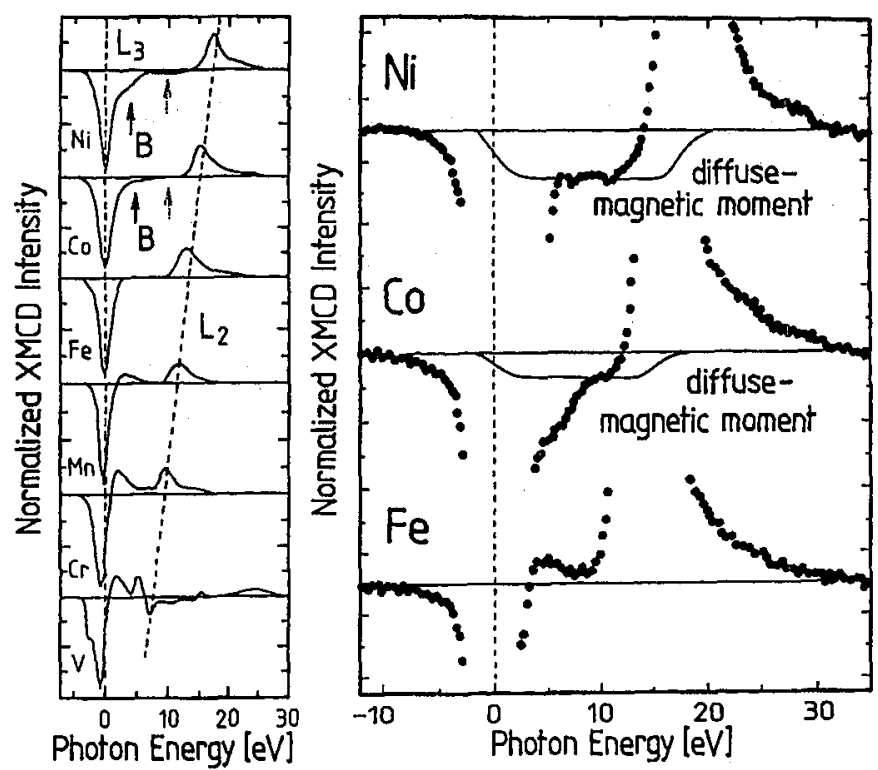

Fig. 9. XMCD spectra of $\mathrm{Ni}$, Co and Fe thin films; data for $\mathrm{Mn}, \mathrm{Cr}$ and $\mathrm{V}$ were obtained on ultrathin films grown on either $\mathrm{Fe}$ or Co substrate. The figure on the right-hand side shows the spectra in enlarged scale. Reproduced with permission from Ref. [19]. Copyright by the American Institute of Physics.

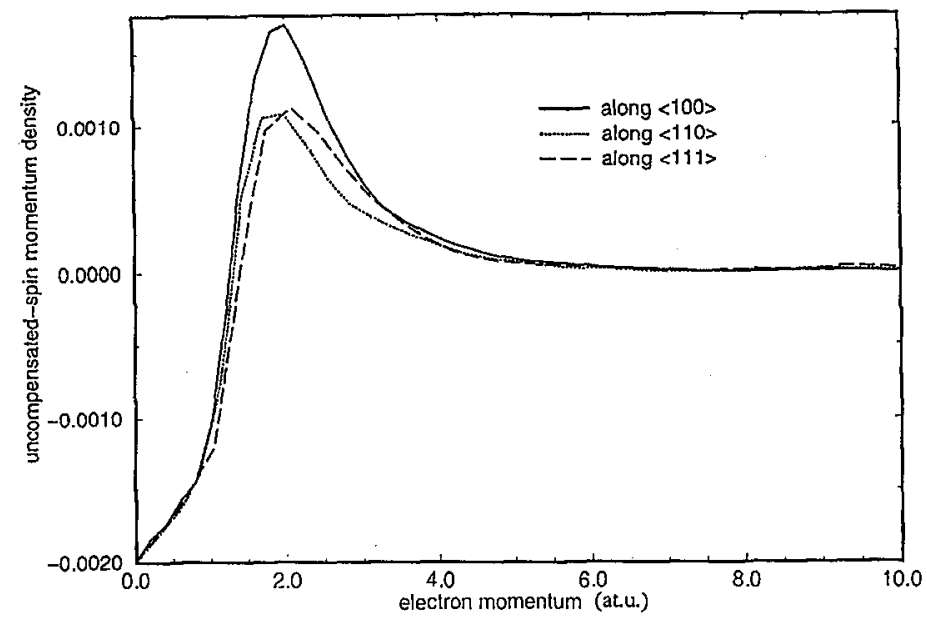

Fig. 10. Reconstruction of the electron momentum density for the spin-uncompensated electrons in $\mathrm{Fe}$ (after [25]); $\alpha=0.5$, modified nonuniform prior.

is the property of the Compton scattering that it is particularly sensitive to the momentum distribution of the outermost electrons. Therefore any polarization of the conduction band should be clearly visible in the magnetic Compton studies. Indeed, it is the case. For the aforementioned reasons, the conduction electrons should show up mainly in the low momentum region. The reconstruction of the 
electron momentum density for the electrons with uncompensated spins in iron, carried out by the maximum entropy method for the experimental data of [24] is shown in Fig. 10 [25]. The negative magnetization is clearly seen at low momenta.

Of course, there is no reason to think that the polarization of the conduction band must be always negative, i.e. antiparallel to the resultant moment. An example is brought by recent magnetic Compton scattering studies on hcp Sm for which clearly positive enhancement of the "magnetic" momentum densities was observed [26] at low electron momenta. The estimated positive magnetic moment of the conduction electrons is in this case as high as $17(3) \%$ of the $4 f$ spin moment.

It is also interesting that the Compton scattering, which is carried out usually at high energies, senses the spin moment only [27]. Therefore, by comparing the results of magnetic Compton scattering with the results obtained by other techniques one determines again spin and orbital moments independently.

\section{Summary}

We have tried to sketch some of the information which is available now by means of X-ray and neutron techniques. Certainly many of the important and interesting papers showing how neutrons and X-rays can be used complementarily have been omitted. The limited time and space did not allow for reviewing very interesting studies of e.g. magnetic correlations in UP [28, 29], which exhibit two length scales at the phase transition point [30], or antiferromagnetic correlations across the spacer in $\mathrm{Ni}_{80} \mathrm{Fe}_{20} / \mathrm{Ag}$ multilayers [31]. Very important achievements of the neutron scattering in the field of the crystal and spin lattice dynamics, where neutrons have no good X-ray counterpart, were not even touched. The interested reader may be referred to e.g. recent reviews published in Physics World [32]. Very recent review of the achievements of the X-ray magnetic scattering can be found in Ref. [33]. We tried to show how subtle physical phenomena can be discovered and described when both techniques are in use. Whether this goal was achieved must be left to the judgement of the readers.

\section{References}

[1] F. de Bergevin, M. Brunel, Phys. Lett. A 39, 141 (1972).

[2] K. Namikawa, M. Ando, T. Nakajima, H. Kawata, J. Phys. Soc. Jpn. 54, 4099 (1985).

[3] M. Blume, J. Appl. Phys. 57, 3615 (1985).

[4] V. Fernandez, C. Vettier, F. de Bergevin, C. Gilles, W. Neubeck, Phys. Rev. B $57,7870(1998)$.

[5] E.D. Isaacs, D.B. McWhan, R.N. Keliman, D.J. Bishop, G.E. Ice, P. Zschack, B.D. Gaulin, T.E. Mason, J.D. Garett, W.J.L. Buyers, Phys. Rev. Lett. 65, 3185 (1990).

[6] A. Vigliante, M.J. Christensen, J.P. Hill, G. Hegelsen, S.A. Sorensen, D.F. McMorrow, Doon Gibbs, R.C.C. Ward, M.R. Wells, Phys. Rev. B 57, 5941 (1998).

[7] J.P. Goff, C. Bryn-Jacobsen, D.F. McMorrow, G.J. McIntyre, J.A. Simpson, R.C.C. Ward, M.R. Wells, Phys. Rev. B 57, 5933 (1998). 
[8] Neutron and Synchrotron Radiation for Condensed Matter Studies, Eds. J. Baruchel, J.L. Hodeau, M.S. Lehmann, J.R. Regnard, C. Schlenker, Vol. I-III, Les Editions de Physique-Springer Verlag, Berlin 1993, 1994.

[9] M. Acet, E.F. Wassermann, K. Andersen, A. Murani, O. Schärpf, Europhys. Lett. 40, 93 (1997).

[10] M. Acet, H. Zähres, E.F. Wasserman, W. Pepperhof, Phys. Rev. B 49, 6012 (1994).

[11] V.L. Moruzzi, P. Marcus, J. Kübler, Phys. Rev. B 39, 6957 (1989).

[12] S. Andrieu, M. Finazzi, F. Yubero, H.M. Fisher, Ph. Arcade, F. Chevrier, L. Hennet, K. Hricovini, G. Krill, M. Piecuch, Europhys. Lett. 38, 459 (1997).

[13] W.L. O'Brien, B.P. Tonner, Phys. Rev. B 50, 2963 (1994).

[14] A. Zunger, in Statics and Dynamics of Alloy Phase Transitions, Eds. P.E.A. Turchi, A. Gonis, Vol. B 319, NATO ASI Series, Kluwer, Dordrecht 1994, p. 361 .

[15] X. Jiang, G.E. Ice, C.J. Sparks, L. Robertson, P. Zschack, Phys. Rev. B 54, 3211 (1996).

[16] S. Brauer, G.B. Stephenson, M. Sutton, R. Brüning, E. Dufresne, S.G.J. Mochrie, G. Grübel, J. Als-Nielsen, D.L. Abernathy, Phys. Rev. Lett. 74, 2010 (1995).

[17] G. Grübel, D. Abernathy, G.B. Stephenson, S. Brauer, I. McNulty, S.G.J. Mochrie, B. McClain, A. Sandy, M. Sutton, E. Dufresne, I.K. Robinson, R. Fleming, R. Pindak, S. Dierker, ESRF Newsletter 23, 14 (1995).

[18] G. Schütz, P. Fischer, E. Goering, K. Attenkofer, D. Ahlers, W. Rössl, Synchrotron Radiat. News 10, 13 (1997).

[19] W.L. O'Brien, B.P. Tonner, G.R. Harp, S.S.P. Parkin, J. Appl. Phys. 76, 6462 (1994).

[20] O. Eriksson, A.M. Boring, R.C. Alberts, G.W. Fernando, B.R. Cooper, Phys. Rev. $B$ 45, 2868 (1992).

[21] B. van Laar, F. Maniawski, S. Kaprzyk, L. Dobrzyński, Nukleonika 25, 835 (1980).

[22] L. Dobrzyński, R. Papoular, M. Sakata, J. Phys. Soc. Jpn. 1, 255 (1996).

[23] Compton Scattering, Ed. B. Williams, McGraw-Hill, 1977.

[24] Y. Tanaka, N. Sakai, Y. Kubo, H. Kawata, Phys. Rev. Lett. 70, 1537 (1993).

[25] L. Dobrzyński, E. Żukowski, to be published in J. Phys. C, Condens. Matter.

[26] H. Adachi, H. Ino, A. Koizumi, N. Sakai, Y. Tanaka, H. Kawata, Phys. Rev. B 56, R5774 (1997).

[27] P. Carra, M. Fabrizio, G. Santoro, B.T. Thole, Phys. Rev. B 53, R5994 (1996).

[28] A. Stunault, S. Langridge, C. Vettier, D. Gibbs, N. Bernhoeft, Phys. Rev. B 55, 423 (1997).

[29] D. Wermeille, A. Stunault, C. Vettier, R. Currat, N. Bernhoeft, to be published.

[30] R.A. Cowley, Phys. Scr. Vol. T 66, 24 (1996).

[31] J.A. Bocherchers, P.M. Gehring, R.W. Erwin, J.F. Ankner, C.F. Majkrzak, T.L. Hylton, K.R. Coffey, M.A. Parker, J.K. Howard, Phys. Rev. B 54, 9870 (1996).

[32] J. Finney, U. Steigenberger, Physics World, December 1997, p. 27; G. Aeppli, S. Hayden, T. Perring, ibid., p. 33.

[33] J.P. Hill, Report BNL-65141, 1998. 